ENSAYOS

\title{
EL DESARROLLO DE LA COMPETENCIA COMUNICATIVA INTERCULTURAL EN LA FORMACION INICIAL DOCENTE
}

The intercultural communicative competence development into the initial teacher training

\section{Manuel Rubio M.}

Tesista del doctorado en lingüística de la PUCV*. Curicó 88, Depto. 713, Santiago. mrubio29@yahoo.es

\section{Resumen}

El propósito de este artículo es presentar una reflexión sobre los desafíos planteados a la formación inicial docente en una sociedad caracterizada por una serie de tendencias que contribuyen a la coexistencia de visiones y estilos diferentes de vida. Se utiliza el concepto de Competencia Comunicativa Intercultural propuesto por Byram (1997) como un marco desde el cual configurar la formación de un educador comprometido con el desarrollo de una ciudadanía compleja (Bolívar 2004). Para contextualizar esta reflexión se describe la matriz cultural desde la cual se ha inscrito tradicionalmente la labor de la escuela y los cambios que supone la implementación de programas de educación intercultural.

Palabras clave: formación docente, educación intercultural, ciudadanía, cultura, comunicación, rol docente, escuela.

\begin{abstract}
The aim of this paper is to present a reflection about challenges raised to initial teacher training into a changing society, which is characterized by current tendencies demanding the different vision and ways of life coexistence. The Intercultural Communicative Competence concept, developed by Byram (1997), is used as a framework for thinking about teacher compromise with the improving a complex citizenship (Bolivar 2004). As a context, the cultural matrix into which the school has done its mission and the changes meaning by the intercultural education programs are described.
\end{abstract}

Key words: teacher training, intercultural education, citizenship, culture, communication, teacher role, school.

\footnotetext{
* Becario MECESUP/PUCV.
} 


\section{INTRODUCCION}

Cuando se pretende configurar un currículo de formación que responda a las necesidades sociales y culturales que enfrentarán los futuros docentes, es necesario partir por hacer referencias a dos tendencias que caracterizan la situación actual del país: la globalización y la postmodernidad.

La globalización hace referencia a la creciente internalización de los mercados y los medios de comunicación, de tal manera que las barreras nacionales y el poder de los estados nacionales se relativiza. La limitación de las capacidades transformadoras de los gobiernos nacionales se explica, por un lado, debido a que el poder económico y financiero reside en centros de poder transnacionales que imponen políticas orientadas al equilibrio macroestructural y a la reducción del rol proteccionista del Estado y, por otro, porque la construcción de sentidos e interpretaciones de la realidad está mediada por el acceso a un caudal de información dominado por las grandes agencias de noticias y publicidad y, al mismo tiempo, por el acceso a una variedad de conocimientos y estilos de vida socializados mediante Internet.

En la era de la globalización el conocimiento ha devenido en una mercancía, es decir, un valor que se intercambia y genera ganancias, puesto que permite el desarrollo tecnológico, la revolución de las comunicaciones y la producción de variados servicios a mercados cada vez más segmentados y exigentes.

Por su parte, la postmodernidad expresa una reflexión que pone el acento en el cuestionamiento de la razón, la idea de progreso y los grandes relatos que orientaron las transformaciones sociales durante el siglo XX. El pensamiento postmoderno es complejo, puesto que no refleja una postura uniforme frente al proyecto de la modernidad; para algunos estamos frente a su fracaso y reemplazo por una nueva matriz cultural; para otros corresponde a un punto de inflexión en que la modernidad se evalúa a sí misma y enriquece críticamente sus postulados básicos: emancipación, expansión, renovación y democracia (García Canclini 2001; Brunner 1998).

La postmodernidad como estilo cultural celebra la diversidad, la fragmentación, la incertidumbre, la inmediatez en la producción de una realidad que se entiende básicamente como interpretación simbólica.

Sin lugar a duda, ambas tendencias contribuyen a una pluralidad y coexistencia de visiones y estilos de vida diferentes que desafían la misión social e integradora de la escuela. Congruentemente, tal situación exige importantes transformaciones en las prácticas pedagógicas y en la concepción del currículum para dar cabida a una educación de carácter intercultural que potencie este contacto, brindando la posibilidad de comprenderlo, valorarlo y asumirlo como un elemento que, seguramente, impactará en la configuración de relatos que los sujetos tienen de ellos mismos y de las interacciones que desarrollen con otros.

Considerando lo anterior, el objetivo de este trabajo es reflexionar sobre la formación inicial docente en el contexto de una educación intercultural. Para hacerlo, partiremos por describir los supuestos culturales en que se ha inscrito tradicionalmente la labor de la escuela. Posteriormente, revisaremos las prácticas de interculturalidad que se han venido implementando en las últimas décadas, referidas fundamentalmente a la integración de las culturas originarias en escuelas situadas en zonas de alta densidad indígena. Tal revisión posibilitará centrarnos en la identificación de algunos principios que fundamenten 
una acción educativa intercultural enfocada en la constitución de un tipo de ciudadanía pertinente a cambios impulsados por la globalización y el postmodernismo. Finalmente, pasaremos a plantear algunas ideas en torno a lo que debiera ser la potenciación de la competencia comunicativa intercultural en estudiantes de pedagogía básica.

El propósito no es realizar un diseño curricular acabado, sino más bien establecer algunos planteamientos generales que sirvan como primera aproximación a esta problemática que, sin duda, requiere de mayor investigación.

\section{LA EXCLUSION DEL OTRO: EL MITO SOBRE LA HOMOGENEIDAD CULTURAL}

El encuentro de culturas diferentes no es una realidad nueva en América Latina. Desde lo que Larraín (1995) denomina "el trauma de la conquista", se han venido dando una serie de relaciones de convergencia y oposición, sometimiento y resistencia entre grupos étnicos que adscriben a matrices culturales diversas. En primer lugar, fueron los españoles con su cosmovisión medieval, su cristianismo de guerra y radical oposición a la secularización modernizante, quienes se impusieron sobre los diversos pueblos indígenas que poblaban el continente, imponiendo como dominante una racionalidad centrada en el autoritarismo político, el culto mariano y la desvalorización de lo indígena. A pesar del mestizaje racial y cultural que tal encuentro produjo, es la cultura hispana la que se privilegia y se constituye en el ideal de la civilización.

Posteriormente, con los procesos de independencia y conformación de estados nacionales, llevados a cabo por la élite criolla, los países latinoamericanos se abren a la influencia de otros países europeos y, en su afán por superar el retraso económico y social, se concibe la lucha entre civilización y barbarie, la cual se originaría en la deficiencia de nuestra raza. Todo lo que tenemos de mestizo se tilda como negativo y, en consecuencia, se privilegia la inmigración de contingentes europeos, en diversos momentos de la vida republicana, que posibiliten la industria y el progreso, que estaría vedado dada la irracionalidad de lo indígena y mestizo (Larraín 1995, 2001).

Así, en Chile, nuevos componentes étnicos deseados -fundamentalmente alemanes y croatas- se hacen parte de la nacionalidad, signados por una valoración positiva y, además, apoyados por una política pública que le asigna una serie de privilegios. Paralelamente, en diferentes ciclos migratorios se harán parte de la nación personas -no necesariamente deseadas- provenientes del Medio Oriente y España. Estos ciclos migratorios se han visto acrecentados por la globalización, la que ha provocado la llegada al país de una gran cantidad de peruanos, cubanos y ecuatorianos, entre otros.

A lo largo de su historia, América Latina, y como parte de ella Chile, se ha caracterizado por lo que Hopenhayn (2002) denomina "la dialéctica de la negación del otro", que se entiende como la exclusión simbólica y material de grupos étnicos y culturales (indios, negros, mestizos, zambos, campesinos, mujeres y marginales urbanos).

De este modo, las clases dominantes fueron imponiendo una visión que aboga por una supuesta homogeneidad cultural que debe promoverse y salvaguardarse. La estrategia privilegiada ha sido la del "blanqueamiento" (Montecino 1996), el cual ha significado la aculturación, degradación e, incluso, el exterminio de las cosmovisiones indígenas y de quienes las sustentan. 
Según Montecino (1998), la estrategia del "blanqueamiento" corresponde a un proceso de identificación que el mestizo realiza con lo hispano y, no obstante, percibir la diferencia. Dicha estrategia privilegia categorías europeas para definir la realidad social y construir una versión de la identidad que subordine y anule lo indígena. Esta estrategia, sin duda, ha estado presente en las interpretaciones históricas dominantes. De hecho, al observar el relato de dos destacados historiadores: Francisco Encina y Jaime Eyzaguirre, esta autora constata que:

La lectura de los relatos de Encina y de Eyzaguirre, teje los hilos de una "chilenidad" que se mira como homogénea. La prevalencia de lo "blanco" sobre lo indio y lo negro es el hito fundamental de su mitología de origen. Origen que culminaría en una "unidad", en una "igualdad", dada por rasgos biológicos (Encina) y culturales (Eyzaguirre) que nos hacen ser "blancos", europeos. Para un autor (Encina), sin negar el peso del mestizaje, lo importante es que su producto es más europeo que mapuche; para el otro (Eyzaguirre), los criollos son los fundadores de la identidad. Para ambos, el sujeto popular, el pobre, el desamparado será el "remanente", el espejo de una realidad histórica en donde se depositó lo indígena en la sociedad nacional (Montecino 1996: 130).

En este contexto, la escuela se conformó en los orígenes de la República como un aparato ideológico orientado a la reproducción de la cultura dominante y el sometimiento de los mestizos, indígenas y negros. Su función ha sido fundamentalmente la homogeneización nacional, la asimilación cultural y la generación de un sentimiento de nacionalidad. En esta perspectiva, su misión ha sido promover la civilización y el desarrollo, eliminando lo barbárico que caracterizaría lo latinoamericano mediante el disciplinamiento corporal, espacial, cronológico, moral y estético (Bolívar 2004; Larraín 1995; Egaña 1996).

Nuestros Estados vieron a la educación como la institución privilegiada para propiciar la aculturación de los indígenas latinoamericanos y su asimilación al cauce de la sociedad criolla englobante, como resultado de su aprendizaje de las formas hegemónicas de actuar, sentir, ser y pensar... De más está decir que, en aras de la construcción de una identidad y cultura nacionales, el proyecto uniformizador suponía un desaprendizaje paulatino, pero seguro, de las instituciones y expresiones culturales y lingüísticas propias (López 2001: 2).

En Chile, la misión civilizadora de la escuela se ha fundado en la "dialéctica de la negación del otro" y en la transmisión de una versión de la identidad nacional, fuertemente esencialista, que es una mezcla de glorias del Ejército y un espíritu guerrero (Larraín 1995). Tal versión promueve y celebra la unidad racial del pueblo chileno y sus campañas victoriosas en la guerra de Arauco, el proceso de independencia y en contra de la Confederación Perú-Boliviana durante el siglo XIX (Larraín 2001).

En definitiva, la creciente escolarización ha significado la negación de un hecho sociológico fundacional: la coexistencia de múltiples grupos étnicos con sus respectivas culturas, invisibilizando las marcadas diferencias sociales, económicas, políticas y simbólicas que caracterizan al alumnado; excluyendo visiones alternativas sobre la identidad, la historia y los proyectos de país que se han debatido en diferentes momentos; perpetuando una visión alienada que nos visualiza como una cultura uniforme desde marcos interpretativos fundamentalmente europeos. 
En la realidad que históricamente han configurado las clases dominantes y han transmitido a través de la escuela, pareciera que no hay lugar para la pluralidad, la negociación de sentidos y la toma de conciencia sobre el derecho a ser reconocidos como un "otro" en términos culturales.

\section{LA EDUCACION INTERCULTURAL EN CHILE}

Según López (2001), la noción de interculturalidad en América Latina surge en la década del 80 fuertemente ligada al tema de la defensa y promoción de la identidad y los derechos de los pueblos indígenas. De hecho, en el continente y en Chile diferentes movimientos indígenas se constituyen en actores sociales y políticos que ponen en la discusión pública la exclusión de la cual han sido víctima. De esta forma, plantean un agudo cuestionamiento a la tradición homogeneizante y a la ideología del mestizaje, cuya función principal habría sido la del ocultamiento de la pluralidad étnica y cultural que caracteriza a cada uno de los países del continente.

Esta visibilidad indígena habría servido como fuente de inspiración para el renacimiento étnico de otras comunidades excluidas de la sociedad latinoamericana, como la de los afroamericanos y la de la migración extranjera (López 2001).

En Chile, la institucionalización de la educación intercultural se logró mediante la promulgación de la Ley $\mathrm{N}^{\circ} 19.253$ de 1993 que crea la Corporación Nacional de Desarrollo Indígena (CONADI) y establece normas de protección y fomento para los pueblos mapuche, rapa nui, aymara, quechua, atacameño, colla, alacalufe y yagán. En esta ley se plantea la necesidad de adaptar el sistema escolar a las demandas de protección del patrimonio y de fomento cultural y lingüístico de dichas comunidades. Esta política se ve reforzada con la reforma curricular del sistema educativo nacional que se inicia en 1996, puesto que se comienzan a ejecutar una serie de proyectos financiados por el Mineduc (Donoso Romo 2000).

Inicialmente, de acuerdo con Donoso Romo (2000), la educación intercultural se comienza a desarrollar en zonas rurales con alta concentración indígena, por lo cual podemos suponer que los estudiantes y familias involucradas en estos proyectos pertenecen a una misma etnia y vivencian procesos orientados a la toma de conciencia de su historia y costumbres, así como a la configuración de identidad donde lo étnico juega un rol central. En este sentido, la educación intercultural deviene en la implementación de una política basada en la identidad, en lo que somos y nos diferencia de los otros, que en este caso sería la cultura hegemónica y su modelo homogeneizante.

No obstante, según este mismo autor, el censo de 1992 deja en evidencia que más de la mitad de la población indígena se ha desplazado a las ciudades. En otras palabras, los indígenas chilenos han sido parte de las diferentes oleadas migratorias del campo a la ciudad que caracterizaron el siglo XX y que, sin lugar a dudas, generaron problemas de inserción en un medio cultural distinto y, a veces, adverso y fuertemente excluyente.

La alta concentración de indígenas en comunas urbano-populares planteó el desafío de generar proyectos de educación intercultural diferentes a los implementados en el campo, pues los indígenas convivían en establecimientos educacionales con no-indígenas y experimentaban ahí, a pesar de su autoidentificación como tales, distintos grados de aculturación y condiciones de aceptación para expresar sus particularidades culturales. 
Así, en la realidad urbana, la educación intercultural trasciende a las familias indígenas como destinatarios privilegiados y se convierte en un diálogo entre niños y niñas pertenecientes a distintas adscripciones culturales, siendo, potencialmente al menos, un importante factor de problematización de la propia identidad, el cuestionamiento de estereotipos y prejuicios, y de aceptación del otro en tanto sujeto de derecho. Sin lugar a dudas, la inserción de la educación intercultural en la escuela rural y urbana implica una reformulación de la forma de contar la historia nacional y de aceptar la versión dominante de la identidad nacional.

Sin embargo, la educación intercultural urbana se inserta en un tipo de escuela que ha perdido su reconocimiento social, producto de las tensiones que tiene con la economía de mercado y con los medios de comunicación de masas, teniendo dificultades para "leer" su entorno y adaptarse a las demandas que desde allí se le plantean (Donoso Romo 2000). Esto hace suponer que la adopción de un currículo intercultural pudiera ser una suerte de visión simplificadora y reduccionista de las problemáticas ligadas a la identidad y el pluralismo cultural. En efecto, más que una educación orientada a la comprensión y aceptación de distintas formas de actuar, sentir, ser y pensar se podría estar vivenciando una suerte de "exotismo" o de "paternalismo benevolente" frente a personas que siguen signadas como "los otros" externos a nuestra cultura.

Este panorama de la educación intercultural, especialmente en las escuelas urbanas, tiende a hacerse aún más complejo producto de la migración de extranjeros provenientes de países latinoamericanos, motivada por la búsqueda de mejores condiciones de vida. Gran parte de los niños y las niñas inmigrantes se insertan en escuelas populares, experimentan situaciones de hacinamiento, pobreza y discriminación. Su condición de vida los pone ante la disyuntiva de la asimilación, la acomodación o la separación, en la cual la agresión no está necesariamente exenta.

Finalmente, cabe considerar que en el país existen una serie de movimientos que han tendido a problematizar importantes aspectos de la cultura dominante, cuyo discurso gradualmente podría irse legitimando e impactando en la escuela. En este sentido, las perspectivas de género, del ecologismo y de los derechos humanos han encontrado importante aceptación en la reforma curricular y se hacen presentes en la propuesta de Objetivos Fundamentales Transversales (Magendzo, Donoso y Rodas 1997). Además, ya se han ido instalando en la escuela un planteamiento y diversas prácticas de integración de niños y niñas con necesidades educativas especiales.

Como vemos, la complejidad tiende a acrecentarse imponiendo fuertes desafíos y demandas al rol y las prácticas docentes, lo cual, indudablemente, no puede ser desconocido por las instituciones que trabajan en el ámbito de la formación pedagógica inicial.

\section{EDUCACION INTERCULTURAL Y CIUDADANIA}

La adopción de un enfoque intercultural supone necesariamente un compromiso por promover transformaciones sociales y políticas conducentes al mejoramiento de la calidad de vida de los grupos tradicional y actualmente postergados, los que en Latinoamérica y Chile denota a los pueblos indígenas, mujeres y comunidades pobres (Hopenhayn 2002; López 2001; Donoso Romo 2000), a lo cual habría que agregar grupos étnicos no-indígenas excluidos (por ejemplo, gitanos), comunidades religiosas, inmigrantes, minorías sexuales, 
tribus urbanas, discapacitados, entre otros. Aquellos que Orbe y Spellers (2005) denominan los grupos coculturales, puesto que comparten una posición que los marginaliza y carecen de representación dentro de las estructuras dominantes.

Ahora bien, los miembros de los grupos coculturales pueden configurar diferentes estrategias para convivir y negociar con las estructuras dominantes. Respecto de los resultados esperados, algunos pueden optar por la separación (un rechazo activo a los grupos dominantes y otros grupos que no comparten su visión de mundo), la acomodación (la retención de algunas de sus singularidades demandando la transformación social y apelando al pluralismo) o la asimilación (la eliminación de las características distintivas para calzar en la cultura dominante). Tales alternativas se pueden concretar con diversos grados de asertividad o agresividad en circunstancias específicas o, también, como modos de inserción social individuales o colectivos (Orbe \& Spellers 2005).

En consecuencia, no basta con que el enfoque intercultural adopte un enfoque político que apele por transformaciones de las estructuras opresivas, sino que también debe configurar estrategias que permitan la negociación de conflictos, la aceptación de una serie de normas que faciliten el acuerdo y un compromiso por establecer reglas básicas de convivencia social. En este sentido, entendemos el enfoque intercultural como: "la defensa de un saber, unos valores y unas normas de convivencia comunes, pero enriquecido con las aportaciones de todos y respetuosos de otras diferencias que no atenten contra ellos" (Bolívar 2004: 16).

En otras palabras, demandar por políticas que partan por el reconocimiento de las diferencias culturales y que, al mismo tiempo, fomenten la integración a una unidad cultural mayor que se estructura sobre la base del pluralismo, la libertad de expresión y el respeto por los derechos individuales y colectivos. En definitiva, la confluencia de voluntades para profundizar la democracia en el plano político, social y cultural.

La educación al adoptar un enfoque intercultural debiera asumir el concepto de ciudadanía compleja planteado por Bolívar (2004), el cual fundamentalmente apunta a que los individuos y grupos que conforman la sociedad desarrollen un proceso formativo que les permita la creación de valores y responsabilidades comunes, es decir, hacerse parte de una cultura pública compartida que reconozca y potencie las identidades primarias. Dicha cultura pública no niega la diversidad ni la relega al espacio privado, sino que más bien favorece la coexistencia, evitando así la constitución de guetos. En este sentido, la ciudadanía se entiende como una forma de vinculación social orientada a la constitución de un espacio público común, dinamizado por la dialéctica entre integración y diferencia.

Indudablemente, la adopción de una educación intercultural basada en la ciudadanía compleja debiera conducir al logro de tres actitudes fundamentales. En primer lugar, concebir la identidad como una construcción histórica, flexible, en permanente cambio, evitando cualquier tipo de esencialismo que suponga que se constituyó de una vez y para siempre, por lo cual debe permanecer intocada y ser el reservorio de todo lo bueno. En segundo lugar, abogar por una postura proactiva que se comprometa en acciones que permitan avanzar en la igualdad de oportunidades, la superación de la pobreza y el acceso a servicios de calidad. Finalmente, lograr una apertura crítica a los procesos de globalización en la perspectiva de pensarse a sí mismo como un ciudadano del mundo, es decir, miembro de la humanidad con responsabilidades frente a las generaciones actuales y futuras, solidarios con procesos destinados a mayor justicia en diversos países y partidario de la defensa de la diversidad (Bolívar 2004; Ipiña 1997; Larraín 2001; Hopenhayn 2000). 
En relación con la identidad, cabe indicar que los individuos construyen su identidad de acuerdo con su pertenencia a diferentes grupos, por lo cual cabría hablar de que una persona enactúa múltiples identidades de acuerdo a las situaciones de comunicación en las cuales se inserta. Esto quiere decir que el sentido de sí mismo que posea un individuo se define y reformula en la interacción social (Hecht, Warren, Jung \& Krieger 2005). En definitiva, un sujeto que adscribe a diferentes grupos (por ejemplo, un joven mapuche urbano que vive en una comuna pobre, pertenece a un grupo de raperos y practica una religión evangélica), en la comunicación en situaciones concretas, externaliza un tipo de identidad que podría ser diferente a los rasgos que lo caracterizan en otra situación.

En otras palabras, la educación intercultural basada en el concepto de ciudadanía compleja debe abrirse a reconocer que las categorías culturales que caracterizan a los sujetos concretos que interactúan en el proceso de enseñanza y aprendizaje pueden ser múltiples, en construcción y, a veces, contradictorias, por lo cual el educador debiera ser capaz de reconocer las diferencias intragrupales, evitar visiones unidimensionales y ser capaz de visualizar, también, a cada individuo en su singularidad.

\section{LA FORMACION DE LA COMPETENCIA COMUNICATIVA INTERCULTURAL}

El concepto de competencia comunicativa alude al conocimiento tácito que un sujeto adquiere acerca de la adecuación de los enunciados al contexto situacional en el cual se producen. En este sentido, no importa sólo la gramaticalidad de una expresión lingüística, sino que, además, su pertinencia al contexto y a los propósitos del hablante. En consecuencia, la noción de competencia comunicativa está asociada al uso del lenguaje en una variedad de situaciones y funciones sociales (Johnson 2004; Byram 1997).

Desde la perspectiva de Hyme (citado por Johnson 2004), quien propuso el concepto, la competencia comunicativa integra dos elementos: por un lado, el conocimiento de las reglas gramaticales y las reglas socioculturales de uso; y, por otro, la habilidad para el uso, la cual incluye factores que no son cognitivos, tales como la motivación, la confianza, la cortesía, la concentración, etc.

Cabe aclarar que este concepto no implica la actuación real que el sujeto tenga en una situación comunicativa dada, sino que, insistimos, se refiere al conocimiento y la habilidad que posee para desempeñarse en dicha situación. El hecho de que tal competencia se ponga en acción está sujeto a múltiples variables.

Indudablemente, este concepto se ha enriquecido incorporando la perspectiva textual y pragmática, puesto que una comunicación eficaz supone la construcción de expresiones lingüísticas complejas dotadas de coherencia y cohesión y la capacidad de interpretar las intenciones subyacentes en un enunciado emitido por otros. Si se entiende el lenguaje como acción, indudablemente debemos aceptar que los interlocutores no sólo se adaptan a un contexto determinado, sino que lo crean en el proceso de su interacción (Johnson 2004; Byram 1997).

Una interesante ampliación del concepto de competencia comunicativa es el planteado por Byram (1997) que lo liga a la educación intercultural. Para este autor, la competencia comunicativa intercultural (CCI) es una habilidad para el uso del lenguaje en un contexto en el cual se ponen en juego identidades culturales diferentes, es decir, modos como los participantes en el acto comunicativo se definen a sí mismos y conciben el mundo y su 
actuación en él. En consecuencia, un sujeto competente comunicativa e interculturalmente tiene la capacidad de insertarse en contextos diferentes, familiarizarse con ellos y actuar de modo respetuoso con las diferentes posibilidades de construir identidad que han adoptado otras personas, mostrando la habilidad de intercambiar información de manera efectiva, además de establecer y mantener relaciones humanas positivas. En síntesis, la competencia comunicativa intercultural requiere un acto de voluntad y conciencia en torno a elementos subjetivos que podrían influir en el intercambio entre personas de grupos culturales diferentes.

Indudablemente, un docente que adopte la educación intercultural basada en el concepto de ciudadanía compleja debe potenciar la adquisición de una competencia comunicativa intercultural, pues ella le permitirá actuar asertivamente en la creación de contextos amigables que posibiliten la expresión, la descripción, la aceptación, el cuestionamiento, la búsqueda y la integración de diversos modos de actuar, sentir, ser y pensar por parte de los educandos.

Esta CCI puede ser el fruto de un proceso de formación claramente orientado a su adquisición. En esta línea, Byram (1997) ha formulado un modelo que puede ser útil en términos de visualizar los desafíos que implica su formación y, congruentemente, diseñar intervenciones curriculares con este propósito. Una visión sintética de tal modelo se ofrece en la figura 1.

Figura 1

Modelo para la formación de la CCI propuesto por Byram (1997)

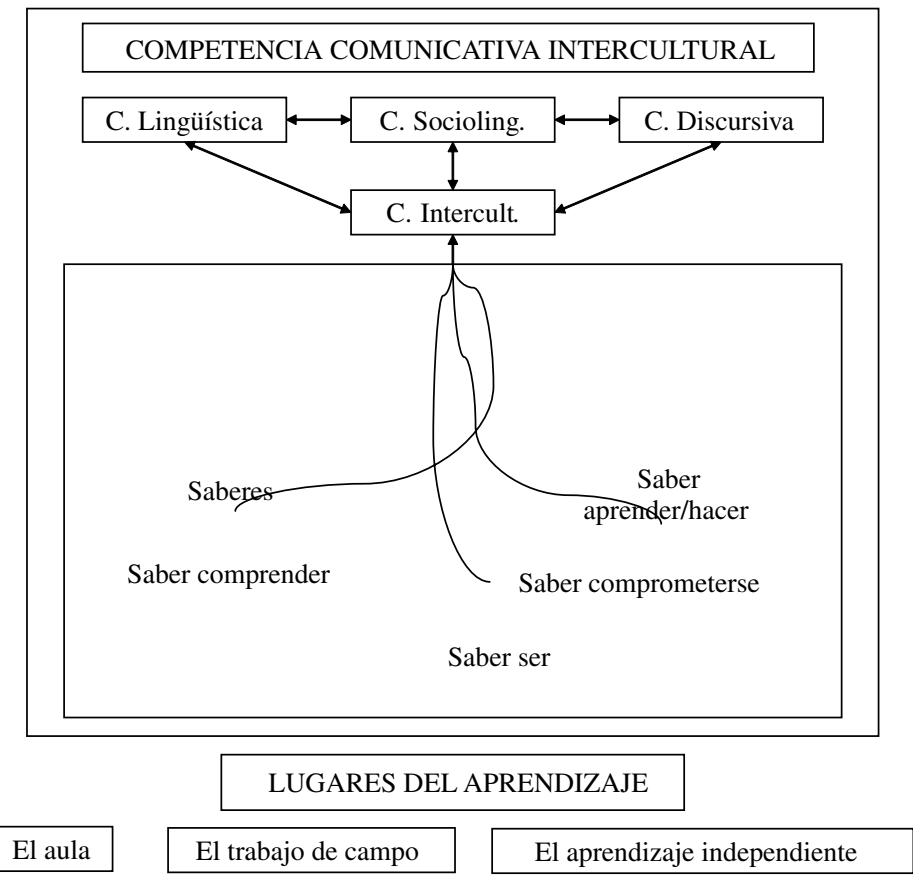


Como se observa en esta figura, el modelo de Byram (1997) supone la generación de diversos saberes para el logro de la CCI, los que se adquieren en diversos lugares de aprendizaje. En efecto, se aboga por una variedad de experiencias tanto dentro como fuera de la sala de clase que permitan el contacto con culturas distintas a la propia. Este contacto puede ser efectivo o virtual; efectivo, mediante la creación de un ambiente que permita la expresión de las singularidades culturales propias de los estudiantes y el acercamiento a determinadas comunidades; virtual, si consideramos las múltiples posibilidades que ofrecen los medios de comunicación (entre ellos, Internet) y el acceso al conocimiento que se difunde mediante revistas especializadas o interlocutores investidos de autoridad para referirse al tema.

Dicha competencia integra a la noción de competencia comunicativa el conocimiento y la habilidad de actuar en contextos culturales diversos o en aquellos donde dos o más culturas entran en contacto, ya sea complementándose u oponiéndose. Las habilidades vinculadas al uso de la lengua, incluidas sus variantes, deben orientarse, de acuerdo con este modelo, al desarrollo de los siguientes saberes (Byram 1997):

a) Actitudes (saber ser). Reconocer los valores, prejuicios y estereotipos que uno posee para dar paso a la curiosidad y apertura a significados, creencias y conductas diferentes. Esto supone una habilidad para "descentrarse" y reconfigurar la propia subjetividad.

b) Conocimientos (saberes). Lograr la comprensión profunda de información relativa a las características singularizadoras de otras culturas, tanto en su dimensión externa (vestidos, códigos lingüísticos, artefactos materiales, patrones paralingüísticos) como interna (creencias, normas, valores, etc.).

c) Habilidades para interpretar y relacionar (saber comprender). Informarse sobre documentación que exponga la visión propia de tales grupos respecto de la historia, identificando situaciones de adaptación y resistencia, siendo capaz de articular una visión comprensiva entre diferentes interpretaciones frente a un mismo fenómeno.

d) Habilidades para descubrir e interactuar (saber aprender/hacer). Percatarse de las interacciones que desarrollan individuos de una misma cultura o de diferentes culturas para poder obtener significados y connotaciones sobre fenómenos propios de la vida cotidiana, la participación social y la configuración de proyectos de vida.

e) Conciencia cultural crítica/educación política. Asumir una postura política que oriente a la acción transformadora basada en el fomento y defensa de los derechos humanos reconocidos por la legislación internacional y nacional.

\section{ALGUNAS PROPUESTAS PARA LA FORMACION DE LA COMPETENCIA COMUNICATIVA INTERCULTURAL DE LOS ESTUDIANTES DE PEDAGOGIA}

De acuerdo a lo que hemos expuesto en los apartados anteriores, la formación de la CCI supone, necesariamente, un cuestionamiento a las prácticas pedagógicas y comunicativas que se dan en la escuela. Esta institución tradicionalmente ha sido un espacio de autoritarismo, disciplinamiento, homogeneización y exclusión.

En efecto, la escuela moderna ha sido un espacio aislado de la comunidad en la cual se inserta y sistemáticamente ha puesto barreras a la expresión de las diferencias 
culturales. Al asumir una misión civilizadora, se hizo parte de una ideología orientada al "blanqueamiento" de la sociedad chilena y a la adopción de una identidad uniforme y excluyente (Egaña 1996; Larraín 2001).

En consecuencia, la formación de la CCI en la formación inicial docente debe partir por un conocimiento del desarrollo histórico de la escuela en nuestro país, las formas opresivas en que se concretó su misión civilizadora y su aporte a la configuración de la versión dominante de la identidad nacional.

Paralelamente, una estrategia orientada a la formación de la CCI debe contribuir a relativizar los conceptos de identidad que manejan los estudiantes, especialmente aquellos referidos a asignarle un valor esencialista que, erróneamente, concibe la identidad como algo que se mantiene inalterado en el tiempo. Para tal efecto, nos parece importante el concepto de hibridación, entendido como un conjunto de "procesos socioculturales en los que estructuras o prácticas discretas, que existían en forma separada, se combinan para generar nuevas estructuras, objetos y prácticas" (García Canclini 2001: 14).

Como se puede deducir de esta definición, el concepto de hibridación no apunta a la esterilidad del cruce entre culturas, sino, por el contrario, a la fertilidad, al enriquecimiento, a la creatividad individual y colectiva que potencia la creación en diferentes esferas de la vida social: la vida cotidiana, la literatura, la música, la tecnología, etc.

En la formación inicial docente el concepto de hibridación se puede utilizar en dos sentidos. El primero se vincula con el estudio de los procesos históricos de mezclas que desde la conquista española se vienen registrando en el país y las formas como tal mezcla ha sido tensionada y transformada por la dinámica de resistencia e integración de los pueblos originarios, la llegada de inmigrantes en diversos momentos, la adopción de estilos de vida foráneos, la emergencia de nuevos actores sociales y el quehacer de las vanguardias artísticas, entre otros. El segundo se relaciona con la experimentación personal de vivencias culturales opuestas que contribuyan a un enriquecimiento de la propia subjetividad mediante la adopción de perspectivas heterodoxas para interpretar los fenómenos que cruzan la convivencia diaria en la comunidad de origen, en la escuela y el país.

A modo de ilustración de este último aspecto, como una actividad de aprendizaje se puede solicitar a los estudiantes que se inserten de manera relativamente permanente en espacios culturales diferentes a los propios, mediante la puesta en práctica de una indagación simple inspirada en la investigación etnográfica (Spradley 1980), lo cual supondría que ellos se hagan participantes regulares de un espacio desconocido, por ejemplo, el mundo de los punkies a través de la asistencia a tocatas. Dicha actividad implicaría observar la actividad de los asistentes, su aspecto físico, las características del ambiente, los modos de relación, el tipo de comportamiento, registrando datos e impresiones de tal manera que un análisis inicial les permitiera formular un par de preguntas de investigación que posibiliten enfocar sus observaciones, tomar contacto para conversar con miembros de esta tribu urbana e ir realizando un análisis cada vez más selectivo. Este proceso, para que efectivamente logre un importante grado de hibridación consciente del sujeto, debiera implicar que los estudiantes expongan sus hallazgos, identifiquen aspectos positivos y problemáticos de la actividad observada y puedan desarrollar una introspección que les permita percatarse de las cogniciones y emociones que van experimentando y, eventualmente, transformando. Esto, indudablemente, en un clima de diálogo que permita la expresión libre, la confianza y el apoyo mutuo entre los estudiantes. 
Asimismo, la literatura y el arte pueden ser un importante apoyo para conocer la perspectiva de personas que, culturalmente, tienen visiones, proyectos y estilos de vida opuestos a los propios. Los diversos géneros literarios ofrecen la posibilidad de una aproximación empática a una realidad que uno no vive directamente, pero sí sustitutivamente al ponerse en el lugar de algunos personajes y experimentar sus emociones. Leer literatura es un medio de crecimiento interior, de ampliar el propio mundo y distanciarse de sí mismo para observar la realidad propia. El arte en su multivocidad permite interpretaciones diversas, mediadas cultural y subjetivamente. La investigación sobre la recepción estética por diferentes grupos es una oportunidad de comprender los filtros sociales y biográficos que condicionan la construcción del significado sobre fenómenos de diversa índole.

Otra importante herramienta es el estudio de casos simples, es decir, una narración breve que presente una situación que podría entenderse como una comunicación intercultural poco efectiva. Esta puede referirse sólo a la interacción entre los miembros de un grupo como al contacto entre miembros de grupos distintos. En ambos casos, se pueden manipular variables que integren diversas dimensiones que contribuyen a configuran los roles que una persona juega en diversas situaciones: género, edad, etnia, orientación sexual, etc. Haciéndolo, se puede clarificar lo que significa entender la identidad como enactuación.

Sin lugar a dudas, técnicas para trabajar la potenciación de la CCI hay muchas y, mediante la creatividad de los docentes, se pueden formular herramientas prácticas diversas e interesantes. Lo importante es adoptar una metodología, entendida como una concepción conceptual y operativa que orienta el quehacer didáctico con los estudiantes de pedagogía, que ponga el acento en los procesos de toma de conciencia, de aprendizaje cooperativo y búsqueda de alternativas de comprensión por parte de ellos.

Dicha metodología apunta a la generación de conocimiento desde la experiencia real o simulada, es decir, debiera considerar a la persona en su integridad, puesto que una persona experimenta una situación desde su ser corporal, cognitivo, emocional, social y espiritual, sea o no consciente de ello. En consecuencia, es preciso dar cabida a la experimentación de vivencias que conecten al sujeto con dimensiones a las que, generalmente, no les presta atención, haciendo un esfuerzo de autoobservación de sí mismo y de los otros. La reiteración de preguntas sencillas en el curso del proceso puede dirigir la atención a determinado aspecto, por ejemplo, al iniciar el diálogo con alguien distinto de mí, ¿cómo estaba mi cuerpo?, ¿en qué medida cambió mi respiración?, ¿qué reacciones esperaba?, etc.

Para que la experiencia devenga en una fuente de conocimiento es necesario conceptualizar este proceso como un diálogo dividido en diferentes momentos, los cuales esquemáticamente se podrían describir como la creación de condiciones para que cada uno de los participantes pueda, individual y cooperativamente:

a) Relatar la experiencia tratando de abarcar las diversas dimensiones que la constituyen, enfocando la atención en aspectos que tradicionalmente permanecen ocultos, como por ejemplo, la propia corporalidad, las intuiciones, las emociones.

b) Objetivar los marcos culturales propios desde los cuales se percibe y asigna significado a la acción propia y de los otros. En otras palabras, evidenciar discursivamente los modos de representación de la propia cultura y de la cultura ajena.

c) Problematizar los discursos explicitados en la fase anterior, tratando de descubrir presuposiciones, prejuicios, estereotipos, valoraciones, etc., que el docente debiera 
presentar como interrogaciones que posibiliten la apertura de los educandos y la toma de conciencia sobre los efectos que significa mantener tales concepciones.

d) Indagar sobre la posibilidad de configurar y poner en práctica pautas actitudinales y conductuales distintas, que se ubiquen en la perspectiva del encuentro intercultural.

e) Comunicar a otros las reflexiones, cuestionamientos, propuestas que se hayan generado como síntesis de los procesos anteriormente descritos.

Para que dicho proceso realmente permita la potenciación de la CCI de los estudiantes de pedagogía, es necesario que el docente sea un mediador capaz de identificar, poner en debate y favorecer la relativización de posturas contrapuestas, estimulando constantemente en los futuros profesores la capacidad de reflexionar, la habilidad de descentrarse y ponerse en el lugar de los otros. Indudablemente, debe tener un tipo de preparación que le permita aportar con información, datos y experiencias relevantes para enmarcar la reflexión en el ámbito de la valoración de la diversidad humana y el respeto a los derechos humanos.

\section{REFLEXIONES FINALES}

Los cambios experimentados por la sociedad, la incapacidad de la escuela para leer las demandas del entorno, la adscripción a un planteamiento de construcción de ciudadanía basado en los derechos humanos y las oportunidades que ofrece la reforma educacional en marcha en el país desde 1996, demandan el replanteamiento de lo que debiera ser la formación inicial de los docentes.

Dicha formación se debe conectar con un proyecto de construcción de una comunidad social basada en la convivencia, la justicia y la interculturalidad, lo cual indudablemente apunta a la generación de procesos de transformación que derriben el mito de homogeneidad cultural, superen la dialéctica de la negación del otro y valoren la coexistencia de grupos con proyectos y estilos de vida, a veces, radicalmente opuestos. Esto, como hemos visto, implica poner a la escuela en el centro del cuestionamiento y, en consecuencia, también los roles y funciones desempeñados por los docentes.

La formación inicial docente en una perspectiva intercultural requiere problematizar las diversas versiones alternativas que se han generado de la nación, así como la de los grupos y sectores sociales que la conforman. Es necesario superar las visiones esencialistas que se oponen a visualizar a las personas como proyectos de ser cada vez más humanos y las aísla de un tipo de interrelación que, potencialmente, pudiera enriquecerlas integralmente. Las personas enactúan diversas identidades de acuerdo con el contexto a los que se integran, porque ellas mismas están configuradas desde posiciones sociales, de género, etnia, edad, capacidad física, habilidades mentales y orientaciones sexuales diversas entre sí. En este sentido, el planteamiento de una identidad común en la cual se comparten ciertos rasgos relativamente permanentes debe constituirse como un proyecto de futuro, permanente, tensionado por las contradicciones que implica la vida social y las demandas de cambio que activan los procesos de globalización y postmodernismo.

La formación inicial de los docentes debe instalarse sobre la conciencia creciente de que avanzamos hacia un mundo cada vez más global y fragmentario, por lo cual el concepto de ciudadanía compleja cimentada sobre la dialéctica entre integración y 
diferencias, sobre los procesos de hibridación que siguen dándose, debiera conducir la labor de un nuevo tipo de escuela, que valore la heterogeneidad cultural, los esfuerzos de integración y convivencia.

Ser competente comunicativa e interculturalmente supone poseer el conocimiento y la habilidad para utilizar el lenguaje como generador de contextos amigables, basados en la expresión libre, el respeto por los derechos ajenos, la aceptación mutua y la búsqueda por espacios de dignidad. En definitiva, la competencia comunicativa intercultural tiene que entenderse como un acto de voluntad y conciencia por el encuentro entre seres singulares que portan visiones culturales heterogéneas $\mathrm{y}$, muchas veces, contradictorias.

\section{BIBLIOGRAFIA}

Bolívar, A. (2004). Ciudadanía y Escuela Pública en el Contexto de la Diversidad Cultural. Revista Mexicana de Investigación Educativa 9 (20): 15-38.

Brunner, J. J. (1998). Globalización Cultural y Postmodernidad. Santiago: Fondo de Cultura Económica.

Byram, M. (1997). Teaching and Assessing Intercultural Communicative Competence. Clevedon: Multilingual Matters ltd.

Donoso Romo, A. (2000). Consideraciones Prácticas a la Educación Intercultural Urbana desde la Experiencia de la Escuela Tobalaba. Revista Digital Umbral 14.

Egaña, M. L. (1996). Disciplina y espacio escolar en la escuela popular chilena del siglo XIX. Revista de la Academia 2: 10-24.

García Canclini, N. (2001). Culturas híbridas. Estrategias para entrar y salir de la modernidad. Buenos Aires: Editorial Paidós.

Hecht, M., J. Warren, E. Jung \& J. Krieger (2005). The Communication Theory of Identity. Development, Theoretical Perspective, and Future Directions. En Gudykunst, W. Theorizing about Intercultural Communication. California: Sage Publications.

Hopenhayn, M. (2002). El Reto de las Identidades y la Multiculturalidad. Pensar Iberoamérica, Revista de Cultura. $\mathrm{N}^{\circ} 0$ [En línea]. Disponible en: http://www.campus-oei.org/pensariberoamerica/ric00a01.htm

Ipiña, E. (1997). Condiciones y perfil del docente de educación intercultural bilingüe. Revista Iberoamericana de Educación 13: 99-109.

Johnson, M. (2004). A Philosophy of Second Language Acquisition. New York: Vail Ballou Press.

Larraín, J. (1995). Las Teorías de la Identidad Latinoamericana. En Gisi, J.; Larraín, J. \& Sepúlveda F. Cultura e Identidad en América Latina. Santiago: Instituto de Estudios Humanísticos.

Larraín, J. (2001). Identidad Chilena. Santiago: LOM.

López, L. E. (2001). La Cuestión de la Interculturalidad y la Educación Latinoamericana. Santiago: UNESCO. [En linea] Disponible en:

http://innovemos.unesco.cl/medios/Documentos/DocumentosConsulta/eyc/Interculturalidad.pdf

Magendzo, A., P. Donoso \& M. T. Rodas (1997). Los Objetivos Fundamentales Transversales en la Reforma Educativa. Santiago: Editorial Universitaria.

Montecino, S. (1996). Madres y Huachos. Alegorías del Mestizaje Chileno. Santiago: Editorial Sudamericana.

Orbe, M. \& R. Spellers (2005). From the Margins to the Center. Utilizing Co-Cultural Theory in Diverse Contexts. En: Gudykunst, W. Theorizing about Intercultural Communication. California: Sage Publications.

Spradley, J. (1980). Participant Observation. Orlando: Harcourt College Publishers. 